\title{
Time-dependent influence of clinical and demographic characteristics on iv-thrombolysis in the Austrian Stroke Unit Registry
}

\author{
M. Marko ${ }^{1}$, A. Posekany², J. Ferrari ${ }^{3}$, P. Sommer ${ }^{4}$, S. Scharer ${ }^{1}$, S. \\ Szabo $^{1}$, V. Lang ${ }^{3}$, W. Lang ${ }^{3}$, S. Greisenegger ${ }^{1}$. \\ ${ }^{1}$ Medical University of Vienna, Neurology, Vienna, Austria. \\ 2Danube University Krems, Gesundheit Österreich GmbH/BIQG, Austria \\ ${ }^{3} \mathrm{KH}$ Barmherzige Brüder Wien, Neurology, Vienna, Austria. \\ ${ }^{4}$ Krankenanstalt Rudolfstiftung, Neurology, Vienna, Austria.
}

\section{BACKGROUND}

Intravenous tissue plasminogen activator (iv-tPA) is an established treatment in acute ischemic stroke. Even if there were substantial increases in tPA-treatment over the last years, rates are still low in selected subgroups of patients potentially eligible for thrombolysis (1). The aim of our study was to analyse the influence of various clinical and demographic characteristics on ivthrombolysis rates as well as time-dependent changes in these influencing factors in a large cohort of patients with acute ischemic stroke in Austria.

\section{METHODS}

We analysed demographic and clinical characteristics of patients with acute ischemic stroke in the Austrian Stroke Unit Registry (ASUR) from 2006-2017. To analyse time-dependent changes of associations of clinical and demographic factors with tPA-treatment over time, we fitted logistic regression models for the entire observational period including testing for interactions with every ongoing year. Additionally, we compared the model for the 'early period' 2006-2009 against the model for the 'late period' 2010-2017, as those two time-periods showed different behaviour and different relevance of explanatory factors.

\section{RESULTS}

Overall, 92861 patients with acute ischemic stroke were enrolled in the ASUR from 2006-2017; 16332 (17,6\%) of these received iv-tPA. Rates of tPA-treatment increased from $9.8 \%$ in 2006 to $17.0 \%$ in 2010 and $21.9 \%$ in 2017.

In the subgroups of patients $>80$ years, patients with a posterior circulation stroke (PCS), patients without atrial fibrillation (AF) and patients with a prior ischemic stroke we observed a higher increase in thrombolysis rates over time. In patients with an Onset-to-Door-Time $>120$ min we detected a particularly high increase in iv-tPA rates from 2008 onwards. (Figure 1)

For the entire observational period (2006-2017), we observed a significant association with higher odds of receiving iv-tPA over the years, in patients with a pre-morbid mRS 0-2 and in patients treated in a high volume centre (defined as centres treating $>250$ stroke-patients/ year). In patients with posterior circulation stroke (PCS), patients with prior ischemic stroke and patients with peripheral arterial disease (PAD) we observed a significant association with lower odds of receiving ivtPA. Lower odds of receiving iv-tPA were also detected in patients with an Onset-to-Door-Time (ODT) of 120-180, >180minutes and an unknown ODT.

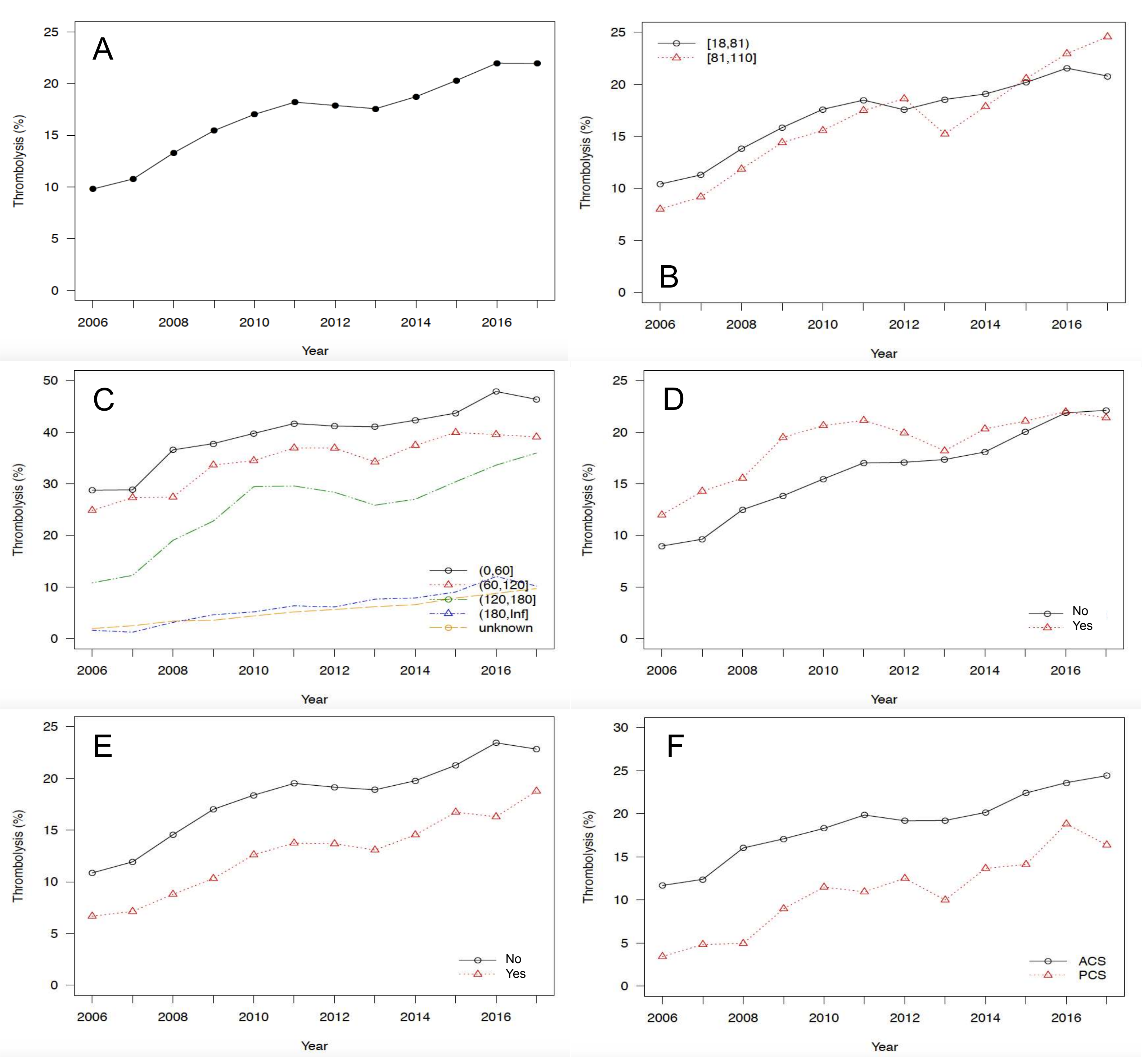

Figure 1: Development of tPA-treatment A: overall, $B$ : in patients $\leq 80$ years and $>80$ years, $C$ : in patients with different Onset-to-Door-Times, D: in patients with atrial fibrillation (AF) and with no documented episode of $A F, E$ : in patients with a prior stroke and with no prior stroke, $F$ : in patients with anterior/posterior circulation stroke (ACS/PCS)

After testing for interactions with every ongoing year we observed decreasing odds of tPA-treatment in patients with atrial fibrillation. In patients $>80$ years, patients with an ODT $>180 \mathrm{~min}$ and patients with a posterior circulation stroke (PCS) we observed increasing odds of tPA-treatment.

Comparing the two consecutive time periods $(2006-2009,2010-2017)$ we observed a significant association of age $>80$ years, stroke severity, premorbid mRS and prior stroke with tPA-treatment for both periods.

In patients with comorbid PAD we detected increasing odds of tPA-treatment over time (2006-2009: OR 0.54 (95\% Cl: 0.41-0.73), $\mathrm{p}=0.006,2010-2017$ OR $0.84(95 \% \mathrm{Cl}: 0.7-1.0), p=n . s)$. Conversely, in patients with atrial fibrillation we observed decreasing odds of tPA-treatment. (2006-2009: OR 0.93 (95\%Cl: 0.78-1.12), p=n.s. , 2010-2017: OR 0.64 (95\%Cl: 0.57-0.72), p<0.001).

\section{CONCLUSIONS}

Overall, rates of tPA-treatment increased over time. Especially in the subgroups of patients with PCS, premorbid PAD, age $>80$ years and an ODT $>180 \mathrm{~min}$ we observed a time-dependent increase in tPA treatment. This might overall reflect an increasing confidence in tPA-use. Conversely, we observed decreasing odds of tPA-treatment in patients with atrial fibrillation over the last 7 years. This might to some extent be due to time-dependent changes in AF-treatment (i.e. increasing use of oral anticoagulants) as well as an overall increasing incidence of AF associated with increasing age in our study population. 